\title{
Expanded porphyrins as third order non-linear optical materials: Some structure-function correlations
}

\author{
SABAPATHI GOKULNATH ${ }^{\mathrm{a}}$ and TAVAREKERE K CHANDRASHEKAR ${ }^{\mathrm{a}, \mathrm{b}, *}$ \\ ${ }^{a}$ Department of Chemistry, Indian Institute of Technology, Kanpur 208016 \\ ${ }^{\mathrm{b}}$ Present address: National Institute for Interdisciplinary Science and Technology (NIIST), \\ Thiruvananthapuram 695019 \\ e-mail: tkc@iitk.ac.in
}

\begin{abstract}
In this paper, the non-linear optical properties of representative core-modified expanded porphyrins have been investigated with an emphasis on the structure-property relationship between the aromaticity and conformational behaviour. It has been shown that the measured two-photon absorption cross section $\left(\sigma^{2}\right)$ values depend on the structure of macrocycle, its aromaticity and the number of $\pi$-electrons in conjugation.
\end{abstract}

Keywords. Expanded porphyrins; conjugation; aromaticity; conformation; TPA cross section.

\section{Introduction}

Expanded porphyrins by virtue of having larger $\pi$ electrons than 18 in conjugation and with bigger cavities are known to have diverse applications. For example, they have been used as anion receptors, sensitizers for PDT, MRI contrasting agents and more recently as non-linear optical materials. ${ }^{1-4}$ The increase in the electron conjugation from $18 \pi$ to 22 , $26,30,34,38,42$ and $54 \pi$ electrons result in changes in the electronic structure which are reflected in spectroscopic and electrochemical properties. ${ }^{5}$ For example, there is a linear red shift of both Soret and Q-bands upon increasing $\pi$-electrons in conjugation resulting in the reduction of HOMO-LUMO gap. ${ }^{6}$ The redox potentials change depending on the structure as well as conformation exhibited by these macrocycles. $^{7}$ It has been shown that the expanded porphyrins exhibit 'normal' structure, 'inverted' structure as well as 'figure-eight' structure depending on the nature of the link at meso carbon bridges as well as the number of $\pi$-electrons in conjugation. ${ }^{6}$ There are examples where the same molecule exhibit planar structure in solution and 'figure-eight' structure in solid state. ${ }^{6 b, 7 a}$ Figure 1 shows the molecular structure exhibited by expanded porphyrins containing six pyrrole/heterocyclic rings linked through either four meso carbon bridges or six meso carbon bridges.

\footnotetext{
*For correspondence
}

An understanding of the structure-function correlations of these expanded porphyrins is an important first step for designing these molecules for specific applications. Recently, we are interested in use of these expanded pophyrins as third order NLO materials. ${ }^{8,9}$ Interaction of the expanded porphyrins with an intense laser light leads to changes in polarization which can be represented as

$$
P=\chi(1) \cdot E+\chi(2) \cdot E E+\chi(3) \cdot E E E+\cdots
$$

where $\chi(2)$ and $\chi(3)$ are the quadratic $\chi(2)$ (firstorder) and $\chi(3)$ cubic (second-order) susceptibilities parameters that determine the magnitude of the second and third order non-linear optical responses. At the molecular level, a similar equation can be written for the light induced molecular dipole moment

$$
P=\alpha \cdot E+\beta \cdot E E+\gamma \cdot E E E+\cdots
$$

with the coefficients $\alpha, \beta$ and $\gamma$ being the linear polarizability, the first (quadratic) hyperpolarizability, and the second (cubic) hyperpolarizability respectively. ${ }^{8 \mathrm{a}, 9 \mathrm{a}}$

The estimation of $\gamma$ can be measured using twophoton absorption method (TPA), ${ }^{9 \mathrm{a}, \mathrm{b}}$ where $\sigma^{2}$ which is a two-photon absorption cross section is measured by the experimental method by the relation: $\sigma^{2}=\left[4 \pi^{2} \hbar \omega / n^{2} c^{2}\right] L^{4} \operatorname{Im}(\gamma){ }^{9 a}$ Thus, the measure of $\sigma^{2}$ by the TPA method gives a direct estimation of $\gamma$. In this paper we wish to relate $\sigma^{2}$ values reported 

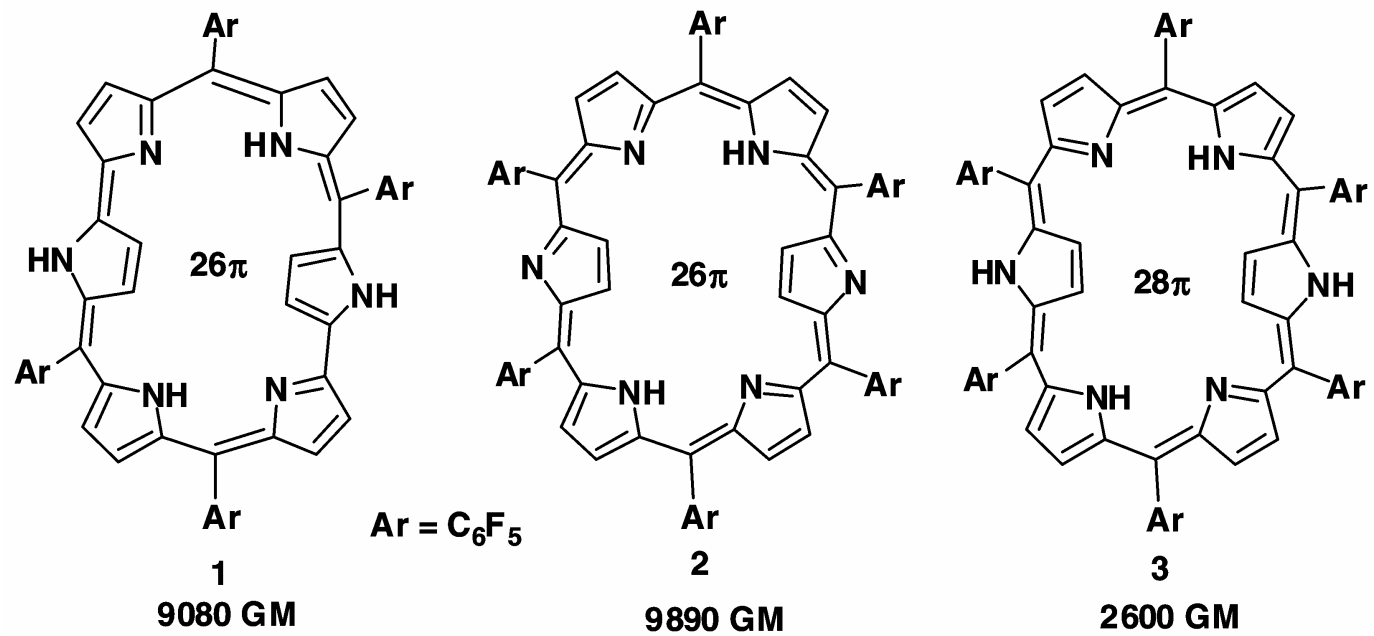

Figure 1. Molecular structure of known hexaphyrins and their $\sigma^{2}$ values.

for various expanded porphyrins to their structure for an understanding of the behaviour of $\sigma^{2}$, specifically to: (a) number of $\pi$-electrons in conjugation, (b) Effect of change of peripheral substituents for electron withdrawing to electron donating groups, (c) changes from 'planar' to figure-eight conformation and vice-versa, and (d) effect of aromaticity (aromatic $v s$ non-aromatic).

\section{Experimental}

The syntheses and characterization of all these expanded porphyrins discussed here was reported earlier by us. ${ }^{10-12}$ Two-photon absorption cross-section measurement were studied by open aperture Z-Scan technique for samples 4-9 by IMRA laser set up. ${ }^{9 a}$ Our femtosecond experimental scheme involves mode locked Coherent Mira titanium: sapphire laser (Model 900) which is pumped by Coherent Verdi frequency doubled $\mathrm{Nd}$ : vanadate laser. The model 900 Mira is tunable from 740 to $900 \mathrm{~nm}$ and its repetition rate is $76 \mathrm{MHz}$. The duration of the pulse is $150 \mathrm{fs}$ as measured by autocorrelation technique. We controlled the average power of the laser pulse in the range of $10-20 \mathrm{~mW}$ by using a half wave plate and a polarizer. In order to reduce the thermal effect which contribute to the $\sigma^{(2)}$ value, we have used a mechanical chopper in the laser path of $\mathrm{Z}$ scan set-up where frequency is as low as $200 \mathrm{~Hz}$. The use of such chopper also reduces the scatter problem seen in some linearly absorbing samples. We used 830 and $860 \mathrm{~nm}$ wavelength here for the measurements. Using a $20 \mathrm{~cm}$ focal length lens, the beam is focused into a $1 \mathrm{~cm}$ long cell filled with sample, where it easily produces GW-level intensity at the focal point of the lens. We scan the sample through the focal point using a motorized translation stage (model ESP 300), which can step with a minimum resolution of $0.1 \mu \mathrm{m}$. This allows a smooth intensity scan for the samples in this wavelength. The transmitted beam, after passing through the sample is focused into the aperture of a UV-enhanced amplified silicon photodetector (Thorlabs DET 210) by using a $7.5 \mathrm{~cm}$ focal length lens. We measure the signal in an oscilloscope (Tektronix TDS 224), which is finally interfaced with the computer using GPIB card. The data were analysed using LabVIEW programme. We obtain the non-linear absorption coefficient $\beta$, by fitting our measured transmittance values to the following formula:

$$
T(z)=1-\beta I_{0} L /\left(2\left(1+z^{2} / z_{0}^{2}\right)\right),
$$

where, $\beta=$ non-linear absorption coefficient, $I_{0}=$ on-axis electric field intensity at the focal point in absence of the sample, $L=$ sample thickness, $z_{0}=$ Rayleigh range $=\pi w_{0}^{2} / \lambda, w_{0}$ is the minimum spot size at the focal point. The $\beta$ values are obtained by curve fitting the measured open-aperture traces with the above equation. After we get the value of $\beta$, the TPA cross-section $\sigma^{(2)}$ (in units of $1 \mathrm{GM}=10^{-50}$ $\mathrm{cm}^{4} \mathrm{~s}$ /photon.molecule), of one solute molecule is given by the following expression:

$$
\sigma^{2}=\beta h v / N c 10^{-3}
$$

where $v$ is the frequency of the incident laser beam, $N$ is Avagadro constant, $c$ is the concentration of the 
compound in dichloromethane solvent. We have taken the known value of $\sigma^{(2)}$ for Rhodamine- $6 \mathrm{G}$ as the reference for calibrating our measurement technique. $^{8 a, 13}$

\section{Results and discussion}

Two-photon absorption (TPA) is a non-linear optical process, occurring under an intense electric field usually generated by pulse lasers with simultaneous absorption of two or more photons by a single chromophore to produce a high-energy excited state. There have been numerous research activities to search for new optical non-linear materials with large two-photon absorption (TPA) cross sections $\left(\sigma^{(2)}\right)$ due to potential applications in a variety of fields including photodynamic therapy, three-dimensional micro-fabrication, optical data storage and optical limiting. ${ }^{2}$ High TPA performance has been achieved only when $\pi$-electronic networks are considerably enlarged either by increasing the $\pi$ conjugation with peripheral substituents ${ }^{9 \mathrm{a}, \mathrm{b}}$ and/or extending the $\pi$-conjugation in cyclic fashion. ${ }^{3,8 a}$

\subsection{Effect of increasing $\pi$-electrons in conjugation}

Addition of increasing number of pyrrole/heterocyclic rings to a porphyrins skeleton leads to expanded porphyrins containing more than $18 \pi$ electrons in conjugation. Interaction of intense laser light with these $\pi$-electrons leads to changes in the polarization thus leading to changes in the observed $\sigma^{(2)}$ values. We have examined expanded porphyrins containing $22 \pi$ through $42 \pi$ electrons in conjugation and evaluated their $\sigma^{(2)}$ values using $\mathrm{Z}$-scan technique.

The $\sigma^{(2)}$ values are depicted along with its molecular structure in figure 2. It is seen that; (a) $\sigma^{(2)}$ values increases linearly with increasing in $\pi$-electrons; (b) $\sigma^{(2)}$ values increases with increase in flexibility. Comparison of the $\sigma^{(2)}$ values for $\mathbf{5}$ and $\mathbf{6}$ illustrates this point. In macrocycle $\mathbf{5}$, six pyrrole/heterocyclic rings are linked through four meso carbon bridges in a (1.1.0.1.1.0) fashion, while in macrocycle 6, six pyrrole/heterocyclic rings are connected through six meso carbon bridges in a (1.1.1.1.1.1) fashion making it more flexible. (c) It should be pointed out here that 8 exhibit 'figure-eight' structure in solid state, while planar structure in solution. Thus, it can be concluded that increase in $\pi$-electrons in conjugation increases the $\sigma^{(2)}$ values in the expanded porphyrins systems.

\subsection{Effect of changing the meso aryl substituents}

The peripheral meso substituents have a marked effect on the $\sigma^{(2)}$ values. Substitution of electron donating and electron withdrawing groups leads to changes in the $\sigma^{(2)}$ values. Substitution of electron donating and electron withdrawing groups leads to changes in the charge transfer direction leading to changes in the $\sigma^{(2)}$ values. For example, the hexaphyrin $6 \mathbf{c}$ containing electron withdrawing group shows a marked increase in the $\left(\sigma^{(2)}\right)$ value as compared to 6a containing electron releasing group at the meso positions. Further, octaphyrin 8a with meso-pentafluorophenyl exhibit reasonably higher value of $81000 \mathrm{GM}$ as compared to $\mathbf{8 c}$ containing mesotrimethoxyphenyl group with $67340 \mathrm{GM}$ (figure 2).

\subsection{Effect of change of heteroatoms}

Changing the inner heterocyclic rings from thiophene to selenophene provides a marked enhancement in TPA values. For example, In hexaphyrin 6a $(2208 \mathrm{GM})$, the replacement of $\mathrm{S}$ with Se leading to 3 -fold increase in the $\left(\sigma^{(2)}\right)$ value as observed for $\mathbf{6 b}$ (7800 GM). Furthermore, the $\left(\sigma^{(2)}\right)$ value of $90600 \mathrm{GM}$ was observed for octaphyrin $\mathbf{8 b}$ with $\mathrm{Se}$ in the core of the macrocycle which is relatively higher when compared to thiophene containing octaphyrin 8a with $81000 \mathrm{GM}$ (figure 2). This effect can be attributed to the heavy atom effect. Substitution of heavier Se for $\mathrm{S}$ leads to changes in the intersystem crossing probably changing the polarization pattern. This is in line with conclusion of Dongho Kim et $a l^{3}$ who have shown that the measured TPA $\left(\sigma^{(2)}\right)$ values in expanded porphyrins depends on singlet and triplet photo dynamics.

\subsection{Effect of aromaticity (aromatic vs non-aromatic)}

Recently, Osuka and Dongho Kim et al reported the photophysical properties along with the measurement of TPA values for 1 (9080 GM), 2 (9890 GM) and $3(2600 \mathrm{GM})$ by an open-aperture Z-scan method. ${ }^{3}$ It was judged from the TPA cross-section $\left(\sigma^{(2)}\right)$ values that reduced antiaromatic hexaphyrin 3 exhibited $\left(\sigma^{(2)}\right)$ value of $2600 \mathrm{GM}$ which is comparatively much lower than its aromatic congeners 1 and 2 (figure 1). Also the $\left(\sigma^{(2)}\right)$ value of $3100 \mathrm{GM}$ for [24]amethyrin $\mathbf{1 0}$ (formally $24 \pi$ hexaphyrin) is comparable to the TPA value obtained for [28] hexaphyrin 3 which is consistent with the reduced aro- 


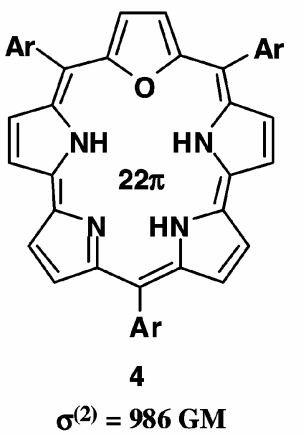<smiles></smiles>

5; $X=S$

$\sigma^{(2)}=7839$ GM

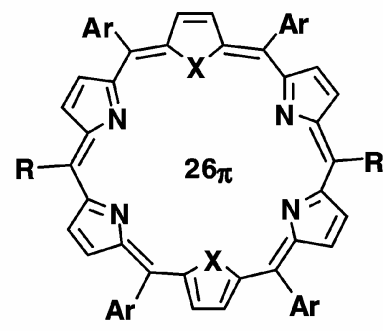

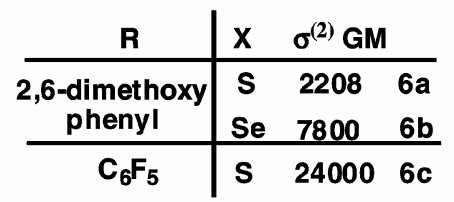

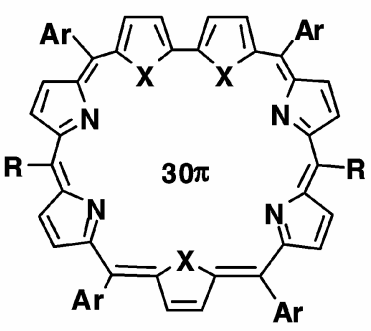

$\mathrm{R}=\mathrm{C}_{6} \mathrm{~F}_{5}$

7; $\mathrm{X}=\mathrm{S}$

$\sigma^{(2)}=68400 \mathrm{GM}$

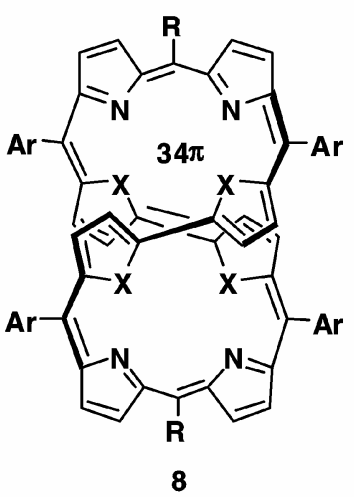

8

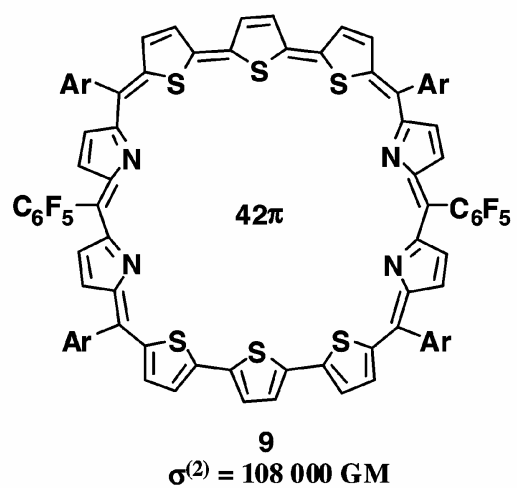

Figure 2. Effect of increasing $\pi$-electron conjugation on $\sigma^{(2)}$ values.

maticity as compared to macrocycles $\mathbf{1 , 2}$ and $\mathbf{6}$. Hence it was concluded that enhancement in the aromatic character leads to increase in the TPA values. Aromaticity of the macrocycle can be related to the planarity of the macrocycle. As the macrocycle become more planar, the $\pi$-electron conjugation pathway is facilitated leading to increase in aromaticity as observed for hexaphyrins by Osuka and cowokers. ${ }^{3 b}$ Furthermore, the $\left(\sigma^{(2)}\right)$ value for [34]octaphyrin 8a was found to be $81000 \mathrm{GM}$ which is considerably higher when compared to its nonaromatic congeners [36]octaphyrin $\mathbf{1 1}$ and $\mathbf{1 2}$ (figure $3)$, thus again reinforcing the dependence of the TPA absorption cross section on the aromaticity.

\subsection{Effect of $\sigma^{(2)}$ values on conformation of the macrocycle}

Expanded porphyrins are constructed by more than six pyrrolic/heterocyclic rings are found to be nonplanar in solid state, whereas they exhibit planar structure in solution and displayed aromaticity. ${ }^{6 b, 8 a}$ The $\sigma^{(2)}$ values depend on the conformation of the expanded porphyrins. Octaphyrins are eight pyrrole/ heterocyclic ring containing macrocycle generally exhibit a 'figure-eight' conformation owing to twist at the meso carbon. This twist results in the loss of aromaticity leads to lower $\sigma^{(2)}$ values. A comparison of $\sigma^{(2)}$ value of $8 \mathbf{a}$ and $\mathbf{1 1}$ reveals the effect of conformation on the $\sigma^{(2)}$ values. For example, 8a shows a planar structure in solution as revealed by spectroscopic and electrochemical studies, whereas 11 exhibit a 'figure-eight' structure both in solution and solid state. Therefore $\mathbf{1 1}$ is expected to show a lower $\sigma^{(2)}$ value owing to its distorted 'figure-eight' conformation which leads to loss of aromaticity. However, in $\mathbf{8}$ the $\sigma^{(2)}$ values are much higher because of the planarity of the macrocycle in solution leading to increase in the aromaticity of the macrocycle and therefore is expected to show higher $\sigma^{(2)}$ values as observed. ${ }^{8 a}$ This observation clearly reveals that the $\sigma^{(2)}$ value depends on the structure and conformation of the expanded porphyrins.

\section{Conclusion}

In this article, an attempt has been made to relate the two-photon absorption cross section $\left(\sigma^{(2)}\right)$ values 


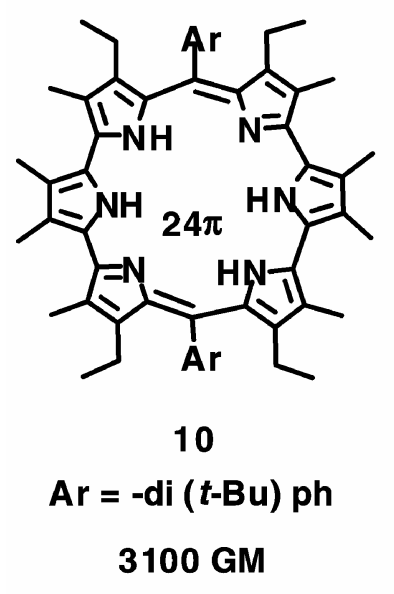

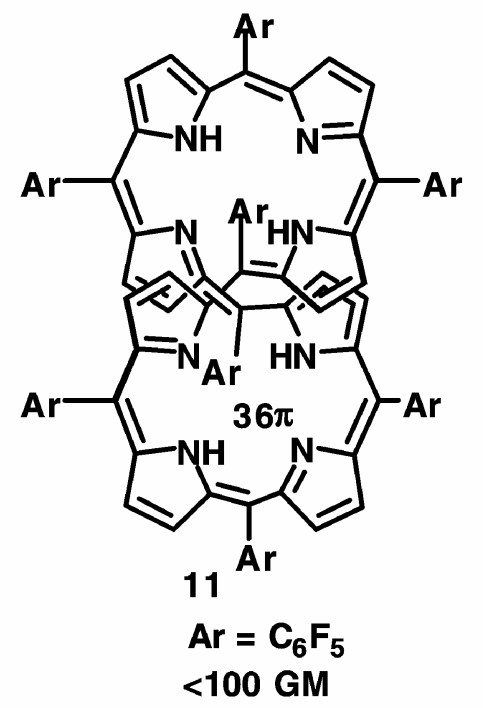

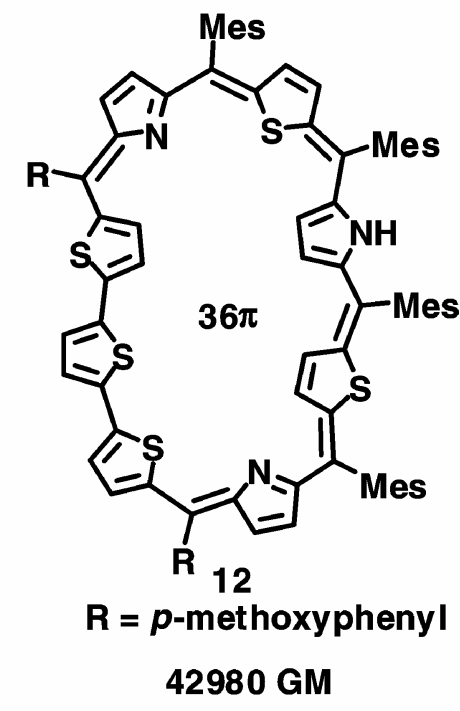

Figure 3. Molecular structures of [24] amethyrin 10, [36]octaphyrin 11 and 12.

measured for a series of expanded porphyrins to their electronic, molecular structure and aromaticity. It has been shown that the increase in $\pi$-electron conjugation and increase in aromaticity leads to higher $\sigma^{(2)}$ values and thus reveal the dependance of $\sigma^{(2)}$ values on the structure as well as aromaticity in the expanded porphyrin systems. These observations reveal that the feasibility of using expanded porphyrins for third-order non-linear applications.

\section{Acknowledgements}

TKC thanks the Department of Science and Techno$\operatorname{logy}$ (DST) for a J C Bose fellowship and the Council of Scientific and Industrial Research (CSIR), Government of India, New Delhi for a research grant. SG thanks CSIR, New Delhi, for a Senior Research Fellowship.

\section{References}

1. (a) For a recent highlight on expanded porphyrins see Sessler J L and Weghorn S J 1997 Expanded, contracted and isomeric porphyrins (Oxford: Elsevier) and references therein; (b) Sessler J L and Weghorn S J 2000 Expanded Porphyrins In Porphyrin handbook (eds) K M Kadish, K M Smith and R Guilard (New York: Academic Press) vol II, chapter 9; (c) Lash T D 2000 Angew. Chem. Int. Ed. 391763

2. (a) Ehrlich J E, Wu X L, Lee I-Y, Hu Z-Y, Rockel H, Marder S R and Perry J W 1997 Opt. Lett. 22 1843; (b) Kohler R H, Cao J, Zipfel W R, Webb W W and Hanson M R 1997 Science 276 2039; (c) Kawata S, Sun H-B, Tanaka T and Takada K 2001 Nature (London) $\mathbf{4 1 2} 697$
3. (a) Ahn T K, Kwon J H, Kim D Y, Cho D W, Jeong D H, Kim S K, Suzuki M, Shimizu S, Osuka A and Kim D 2005 J. Am. Chem. Soc. 127 12856; (b) Yoon Z S, Kwon J H, Yoon M-C, Koh M K, Noh S B, Sessler J L, Lee J T, Seidel D, Aguilar A, Shimizu S, Suzuki M, Osuka A and Kim D $2006 \mathrm{~J}$. Am. Chem. Soc. 12814128

4. (a) Ogawa K, Ohashi A, Kobuke Y, Kamada K and Ohta K 2003 J. Am. Chem. Soc. 125 13356; (b) Drobizhev M, Stepanenko Y, Dzenis Y, Karotki A, Rebane A, Taylor P N and Anderson H L $2004 \mathrm{~J}$. Am. Chem. Soc. 126 15352; (c) Drobizhev M, Stepanenko Y, Dzenis Y, Karotki A, Rebane A, Taylor P N and Anderson H L 2005 J. Phys. Chem. B109 7223

5. For recent studies on TPA cross on porphyrins see (a) Mori S, Kim K S, Yoon Z S, Noh S B, Kim D and Osuka A $2007 \mathrm{~J}$. Am. Chem. Soc. 129 11344; (b) Yoon M-C, Noh S B, Tsuda A, Nakamura Y, Osuka A and Kim D $2007 \mathrm{~J}$. Am. Chem. Soc. 129 10080; (c) Hisaki I, Hiroto S, Kim K S, Noh S B, Kim D, Shinokubo H and Osuka A 2007 Angew. Chem. Int. Ed. 465125

6. (a) Chandrashekar T K and Venkatraman S 2003 Acc. Chem. Res. 36 676; (b) Rath H, Sankar J, PrabhuRaja V, Chandrashekar T K, Joshi B S and Roy R 2005 Chem. Commun. 3343

7. (a) Rath H, Sankar J, PrabhuRaja V, Chandrashekar T K and Joshi B S 2005 Org. Lett. 7 5445; (b) Misra R, Kumar R, Chandrashekar T K and Suresh E 2007 Chem. Commun. 43

8. (a) Rath H, Sankar J, PrabhuRaja V, Chandrashekar T K, Nag A and Goswami D $2005 \mathrm{~J}$. Am. Chem. Soc. 127 11608; (b) Rath H, Sankar J, PrabhuRaja V, Chandrashekar T K, Nag A, Goswami D and Joshi B S 2006 Org. Lett. 82325

9. (a) Misra R, Kumar R, Chandrashekar T K, Suresh C H, Nag A and Goswami D $2006 \mathrm{~J}$. Am. Chem. Soc. 128 16083; (b) Misra R, Kumar R, Chandrashekar T K, Nag A and Goswami D 2006 Org. Lett. 8 629; (c) 
Kumar R, Misra R, Chandrashekar T K, Nag A Goswami D, Suresh E and Suresh C H 2007 Eur. J. Org. Chem. 4552

10. (a) Narayanan S J, Sridevi B, Chandrashekar T K, Vij A and Roy R 1998 Angew. Chem. Int. Ed. 37 3394; (b) Narayanan S J, Sridevi B, Chandrashekar T K, Viji A and Roy R 1999 J. Am. Chem. Soc. 1219053

11. (a) Narayanan S J, Sridevi B and Chandrashekar T K 1999 Org. Lett. 1 587; (b) Rath H, Anand V G, Sankar J, Venkatraman, Chandrashekar T K, Joshi B
S, Khetrapal C L, Schilde U and Senge M O 2003 Org. Lett. 53531

12. (a) Srinivasan A, Anand V G, Narayanan S J, Pushpan S K, Kumar M R Chandrashekar T K Sugiura K-I and Sakata Y 1999 J. Org. Chem. 64 8693; (b) Narayanan S J, Srinivasan A, Sridevi B, Chandrashekar T K, Senge M O, Sugiura K-I and Sakata Y 2000 Eur. J. Org. Chem. 2357

13. Sengupta P, Balaji J, Banerjee S, Philip R, Ravindra K G and Maiti S 2000 J. Chem. Phys. 1129201 